\title{
NOVEL NANOPARTICLES FOR THE ORAL DELIVERY OF LOW MOLECULAR WEIGHT HEPARIN: IN VITRO AND IN VIVO ASSESSMENT
}

\section{LAVANYA NALLAGUNTLA ${ }^{1 *}$, INDIRA MUZIB Y ${ }^{1}$, JITHAN AUKUNURU ${ }^{2,3}$, UMAMAHESH BALEKARI $^{4}$}

${ }^{1}$ Department of Pharmaceutics, Institute of Pharmaceutical Technology, Sri Padmavati Mahila Visvavidhyalayam (Womens university), Tirupathi, Andhra Pradesh, India. ${ }^{2}$ Department of Pharmaceutics, Mother Teresa College of Pharmacy, Hyderabad, Telangana, India. ${ }^{3}$ Aizant Drug Solutions Pvt. Ltd., Hyderabad, Telangana, India. ${ }^{4}$ Department of Pharmacognosy, Faculty of Pharmacy, University College of Pharmaceutical Sciences, Kakatiya University, Warangal, Telangana, India. Email: lavanyanallaguntla@gmail.com

Received: 04 October 2016, Revised and Accepted: 24 October 2016

\section{ABSTRACT}

Objective: The objective of the present study was to prepare and evaluate a novel oral formulation of nanoparticles for the systemic delivery of lowmolecular-weight heparin (LMWH).

Methods: Nanoparticles were prepared by polyelectrolyte complexation method using polymers, i.e., sodium alginate and chitosan (CH). Fourier transform infrared spectroscopy (FTIR), differential scanning calorimetry (DSC), X-ray diffraction (XRD), entrapment efficiency, In vitro release and scanning electron microscopic studies were carried out for nanoparticles. Ex vivo permeation studies were performed with optimized formulation using small intestine of rat and in vivo studies were conducted on rat model.

Results: Entrapment efficiency of LMWH in nanoparticles was found to be 88\%. In vitro release studies demonstrated that the release of LMWH was negligible in the stomach and high in the small intestine. FTIR has indicated that there is no interaction between the ingredients in nanoparticle. DSC and XRD studies confirmed that the amino groups of $\mathrm{CH}$ interacted with the carboxylic groups of alginate. In vitro \% drug release of $95 \%$ was shown by formulation AC5. Ex vivo permeation studies have elucidated that $73 \%$ of LMWH was transported across the epithelium. Nanoparticles have shown enhanced oral bioavailability of LMWH as revealed by 4.5-fold increase in area under the curve of plasma drug concentration-time curve.

Conclusion: The results suggest that the nanoparticles prepared can result in targeted delivery of LMWH into systemic circulation through intestinal and colon routes. Novel nanoparticles thus prepared in this study can be considered as a promising delivery system.

Keywords: Antifactor Xa activity, Chitosan, Differential scanning calorimetry, Sodium alginate, Low-molecular-weight heparin, Oral bioavailability.

(C) 2017 The Authors. Published by Innovare Academic Sciences Pvt Ltd. This is an open access article under the CC BY license (http://creativecommons. org/licenses/by/4. 0/) DOI: http://dx.doi.org/10.22159/ajpcr.2017.v10i2.15514

\section{INTRODUCTION}

Low-molecular-weight heparin (LMWH) is used as an anticoagulant in mostly two common post-surgical complications such as deep vein thrombosis and pulmonary embolism. It is used for long-term dosing through parenteral route and hence has reduced patient compliance. This can be improved by administering LMWH through the oral route. However, its clinical application through oral route is limited because of its large molecular size, high negative charge, instability in gastrointestinal region, and high water solubility. However, several studies addressed oral delivery of LMWH into systemic circulation. Various approaches have been investigated such as microemulsions, nanoparticles, microparticles, pellets, and chemical conjugates [1-5]. Yet, no oral formulation is able to produce good oral bioavailability and is not available in the market yet now. Since LMWH is permeable in the lower small intestine and colon, its penetration enhancement can be conveniently used to produce a market viable oral formulation for LMWH. Subsequently, in the present study, attempts were made to prepare and evaluate $\mathrm{LMWH}$ nanoparticles.

Nanoparticles were selected as they have the following advantages (a) they offer protection against acidic environment and enzymatic degradation, (b) its small size and the bioadhesive polymers used help in prolonging the gastric transit and permeating the mucous membrane. Particle size $(1000 \mathrm{~nm})$ is considered as a crucial parameter for bioadhesion and adsorption through the mucosal membrane. Paracellular and endocytic pathway are the major transport mechanisms for the transport of nanoparticles which majorly depended on particle size [6]. Various types of nanoparticles have been studied earlier [7,8]. Polyelectrolyte complexation (PEC) method is selected for preparation of nanoparticles as these are considered as the emerging delivery systems for the oral delivery of proteins, nucleic acids $[9,10]$, etc. A combination of chitosan $(\mathrm{CH})$ and alginate was used as the polyelectrolyte combination. Previously studies indicated that such nanoparticles are gastric resistant. Further, the natural bioadhesive property of the combination can lead to more residence on the mucosal membrane which in turn can result in significant systemic LMWH.

PEC is formed by spontaneous interaction of oppositely charged polysaccharides in aqueous solution. Polysaccharides are of particular importance due to their desirable biocompatible, biodegradable, hydrophilic, and protective properties. PEC method has the advantage of not using sonication and organic solvents which are harmful for proteins and polysaccharides. Previous investigations confirmed that $\mathrm{CH}$ and alginate were widely used for PEC formation and they have profound applications in drug or gene delivery systems in biomedicine $[11,12]$.

$\mathrm{CH}$ is a linear polysaccharide composed of randomly distributed $\beta$-(1-4)-linked D-glucosamine (deacetylated unit) and N-acetyl-Dglucosamine (acetylated unit) [13]. It is a biocompatible, biodegradable polymer, having inherent mucoadhesive properties with the capacity to open tight junctions in the mucosal membrane. The positive charge of 
$\mathrm{CH}$ forms a strong bond with the negatively charged epithelial lining of gastrointestinal tract. This high interaction helps in opening the tight junction and makes the drug to reach the systemic circulation. However, it is mostly soluble in acidic pH conditions of the stomach, which makes it to lose its mucoadhesive and permeation enhancing properties. To overcome this, in the present study, a complex was formed between $\mathrm{CH}$ and a natural polyanion to improve the physicochemical property of $\mathrm{CH}$. One of the polyanionic polymers that are widely used is sodium alginate (SA).

SA is an anionic polysaccharide distributed widely in the cell walls of brown algae. It is a linear copolymer with homopolymeric blocks of (1-4)-linked $\beta$-D- mannuronate (M) and its C-5 epimer $\alpha$-L-guluronate (G) residues, covalently linked together in different sequences or blocks. The negatively charged carboxylic acid groups of mannuronic and guluronic acid units of alginate interact electrostatically with positively charged amino groups of $\mathrm{CH}$ to form polyelectrolyte. At a $\mathrm{pH}$ of 2.5 , SA is negatively charged. As the $\mathrm{pH}$ increases from 2.5, the number of anionic charges on the SA was increased. While at $\mathrm{pH}<6.5$, $\mathrm{CH}$ is positively charged. The $\mathrm{pH}$ values, i.e. 4.0 for $\mathrm{CH}$ and 6.5 for SA ensures an increased charge density on each polymer and leads to intense cross-linking on mixing [14]. SA is mostly used as polyelectrolyte that forms complex with $\mathrm{CH}$ because the complex formed between the polymers is still biodegradable, biocompatible, and mechanically stronger in acidic $\mathrm{pH}$ conditions where $\mathrm{CH}$ dissolves. A research work has demonstrated that $\mathrm{CH}$ alone was effectively degraded by lysozymes $[15,16]$. However, this enzyme effect on PEC is negligible because of the strong interaction between the two polymeric chains.

In this study, PEC is formed by one-stage process. In this method, one polymer solution of a particular $\mathrm{pH}$ is added dropwise to the other polymer solution of a certain $\mathrm{pH}$ under high shearing conditions. In the present work, LMWH loaded PEC nanoparticles were formulated and it's in vitro release and in vivo absorption are compared with LMWH CH nanoparticles. Such nanoparticles for oral delivery of LMWH were not reported previously

\section{MATERIALS AND METHODS}

\section{Materials}

Enoxaparin sodium (LMWH) was purchased from Bharath Biotech, Hyderabad, India. $\mathrm{CH}$, sodium tripolyphosphate (STPP), dialysis membrane (MW 12,000 Daltons), and cetyl pyridinium chloride were purchased from Sigma-Aldrich Private Limited, Mumbai, India. SA was purchased from SD Fine Chemicals Limited, Gujarat, India. Stachrom Heparin supplied by Diagnostica Stago, Asnieres-sur-Seine, France. All the other chemicals and reagents were of analytical grade and used as supplied. Simulated gastric fluid $\mathrm{pH}$ 1.2, simulated intestinal fluid of $\mathrm{pH} 6.8$ and 7.4 were prepared by referring to official methods as specified in USP (XXV).

\section{Methods}

\section{Reagents used for analysis}

To $1 \mathrm{ml}$ of standard solution, $1 \mathrm{ml}$ of $1 \mathrm{M}$ acetate buffer of pH 5 and $4 \mathrm{ml}$ of cetyl pyridinium chloride solution $(0.1 \%)$ in sodium chloride $(0.94 \%)$ were added and reacted for $1 \mathrm{hr}$. Samples were then analyzed at $500 \mathrm{~nm}$ in ultraviolet (UV)-visible spectrophotometer [17].

\section{Preparation of PEC nanoparticles of alginate and $\mathrm{CH}$}

SA solution was prepared by dissolving SA in reverse osmosis (RO) water. $\mathrm{CH}$ solution was prepared by dissolving $\mathrm{CH}$ in $1 \%$ acetic acid solution. Both the solutions were placed separately on magnetic stirrer at $100 \mathrm{rpm}$ for $1 \mathrm{hr}$. LMWH $(20 \mathrm{mg})$ was then added to SA solution and dissolved. SA solution was adjusted to $\mathrm{pH} 6.5$ and $\mathrm{CH}$ solution to $\mathrm{pH}$ 4.0. SA solution is then slowly added to $\mathrm{CH}$ solution at a flow rate of $1 \mathrm{ml} / \mathrm{s}$ with the help of high-speed homogenizer (Unidrive X1000D Homogenizer drive, CAT scientific laboratory, California) at 10,000 rpm. Alginate in CH PECs was thus formed [10].

\section{Preparation of $\mathrm{CH}$ nanoparticles}

Nanoparticles were prepared by ion gelation of $\mathrm{CH}$ with STPP aqueous solution. First, $\mathrm{CH}$ was dissolved in $1 \%$ solution of acetic acid. LMWH was then added to $\mathrm{CH}$ solution and mixed. Aqueous solution of STPP was added dropwise to $\mathrm{CH}$ solution by magnetic stirring at room temperature using high-speed homogenizer [18].

\section{In vitro characterization of PEC nanoparticles}

PEC nanoparticles and its formulation components were subjected to Fourier transform infrared (FTIR) (Bruker Alpha-E spectrophotometer, Ettlingen, Germany), differential scanning calorimetry (DSC) (automatic thermal analyzer, DSC 910S, TA Instruments, America), X-ray diffraction (XRD) (X'Pert-PRO multipurpose X-ray diffractometer, PANalytical, Tokyo, Japan), scanning electron microscopy (LEO 435 VP, Eindhoven Netherlands), and particle size and zeta potential studies (Zetasizer, Model 3000 HSA, Malvern Instrument, WR14 1XZ, UK).

\section{Entrapment efficiency (EE)}

The EE of LMWH in PEC nanoparticles was performed by an indirect method. The amount of unentrapped LMWH in the nanoparticles suspension was analyzed by taking the supernatant after centrifugation at $15000 \mathrm{rpm}$ for 20 minutes. Then, aliquots of $1 \mathrm{ml}$ of the supernatant was taken and mixed with reagents as discussed previously in the methods and reacted for $1 \mathrm{hr}$. Later, the absorbance of these samples was taken at $500 \mathrm{~nm}$ using UV-visible spectrophotometer. The study was performed in triplicate, and the percentage EE was determined [19].

\section{$\mathrm{EE} \%=\frac{\text { Totalamount of LMWH taken }- \text { FreeLMWHin thesupernatant }}{}$

Totalamount of LMWH taken

\section{Percentage transmittance}

The study was performed 1-3 hrs after the preparation of PECs. RO water was used as the blank and adjusted to $100 \%$ transmittance at $800 \mathrm{~nm}$ using UV-visible spectrophotometer [10]. The \% transmittance of PEC nanoparticles was then measured.

\section{In vitro release study}

The in vitro release study of LMWH from drug loaded PEC nanoparticles was performed using the dialysis membrane method. LMWH PEC nanoparticulate suspension was filled in a dialysis bag (MW-12,000 Da) which was attached to a two-end opened boiling tube [20]. The boiling tube was then dipped in a beaker containing $50 \mathrm{ml}$ of $\mathrm{pH} 1.2$ buffer and placed on a magnetic stirrer for $2 \mathrm{hrs}$ at $37 \pm 0.5^{\circ} \mathrm{C}$ and $50 \mathrm{rpm}$. The medium was then replaced with $\mathrm{pH} 6.8$ buffer and after 3 hrs with $\mathrm{pH} 7.4$ buffer. The release study was performed for $24 \mathrm{hrs}$. Aliquots of $0.5 \mathrm{ml}$ were taken at regular intervals, i.e. 1, 2, 3, 4, 5, 6, 8, 10 up to $24 \mathrm{hrs}$ and analyzed by using UV-visible spectrophotometer at $500 \mathrm{~nm}$. The same experiment was performed for plain $\mathrm{CH}$ nanoparticles and analyzed.

The release data were then fitted to different kinetic models, and the best fit model was determined based on $\mathrm{R}^{2}$ values.

\section{Ex vivo drug release study}

The amount of LMWH transported across the intestinal barrier was measured. Small intestine of male Wistar rat was removed and rinsed with normal saline solution. LMWH loaded PEC nanoparticulate suspension was filled in the membrane, and both ends were tied with a thread separately and fixed to a stand. The membrane was then introduced into $50 \mathrm{ml}$ of $6.8 \mathrm{pH}$ buffer in a beaker. The beaker was then placed on a magnetic stirrer at $37^{\circ} \mathrm{C}$ and $100 \mathrm{rpm}$. Aliquots of $1 \mathrm{ml}$ were taken at intervals of $5,15,30,45,60,90,120$ minutes up to $8 \mathrm{hrs}$. The samples were then analyzed by UV-visible spectrophotometer at $500 \mathrm{~nm}$ by the addition of reagents and reacted for $1 \mathrm{hr}$. Permeation studies were performed in triplicate [21]. 


\section{Stability studies}

The LMWH loaded PEC nanoparticles were studied for stability studies at various temperatures such as $4 \pm 1^{\circ} \mathrm{C}$ and $25 \pm 1^{\circ} \mathrm{C}$ and examined at regular time intervals for any change in particle size and drug content [19].

\section{Oral absorption studies in rats}

All animal studies were approved by the Institutional Ethical Committee, Warangal, registered under CPCSEA, India (IAEC no. $1047 / \mathrm{ac} / 07 / \mathrm{CPCSEA}$ ). Male Wistar rats (weighting $130 \pm 20 \mathrm{~g}$ ) were taken and fastened overnight with free access to water before the administration of formulations. Three groups of animals were taken, each group containing six animals. Group 1 is considered as control which was a plain LMWH solution $(50 \mathrm{mg} / \mathrm{kg})$ and given orally to rats. Formulations such as LMWH loaded plain $\mathrm{CH}$ nanoparticulate suspension and PEC nanoparticles were administered orally to Group 2 and Group 3 rats, respectively, in the same dose. To Group 3, LMWH loaded PEC nanoparticles were administered orally in the same dose. Blood samples were withdrawn from the retro-orbital plexus at 0,1 , $2,3,4$, and 5 up to $8 \mathrm{hrs}$. LMWH was quantified using an anti-factor Xa chromogenic assay. Anti-factor Xa activity versus time profile of LMWH in the plasma was then plotted and compared with that of the oral LMWH solution. Plasma concentrations of the drug were estimated, and different pharmacokinetic parameters were evaluated. Clotting time studies were performed with a plain LMWH solution, LMWH CH nanoparticles, and LMWH PEC nanoparticles on Wistar rats using a laboratory method as specified in reference [22,23]. Briefly, formulations were administered to three groups of animals as mentioned above. At regular time intervals, i.e. 0, 1, 2, 3, 4 up to $8 \mathrm{hrs}$ blood samples were collected and their clotting time was determined by capillary method.

\section{RESULTS}

PECs were frequently prepared by mixing two electrolyte solutions of opposite charges. Various quantities of alginate and $\mathrm{CH}$ were taken at desirable $\mathrm{pH}$ conditions and complexes were prepared (Table 1). The formation of PECs was confirmed by FTIR and DSC studies. From the FTIR spectrum of LMWH loaded PEC nanoparticles; it was observed that the asymmetric and symmetric carboxylate anion ( $\mathrm{COO}^{-}$) stretching was shifted from 1661 to $1638 / \mathrm{cm}$ and from 1450 to $1439 / \mathrm{cm}$, respectively. Furthermore, the absorption band of $\mathrm{CH}$ was shifted from 1582 to $1546 / \mathrm{cm}$ and the broadening of band from 1510 to $1400 / \mathrm{cm}$ was attributed to the overlapping of amide of $\mathrm{CH}$ with carboxyl anion of alginate (Table 2). No characteristic bands of LMWH were observed in the spectrum of nanoparticulate formulation (Fig. 1). DSC confirms the presence of interaction between $\mathrm{CH}$ and alginate. As observed from the thermograms shown in Fig. 2, the endotherms of $\mathrm{CH}$ and SA were exhibited at $111.63^{\circ} \mathrm{C}$ and $130^{\circ} \mathrm{C}$ which indicates the evaporation of absorbed water. An endotherm of PEC nanoparticles was observed at $99.6^{\circ} \mathrm{C}$ which is lower than the endothermic peaks of $\mathrm{CH}$ and SA individually. This indicates that the hydrophilic groups in the PECs were more exposed possibly due to the formation of gaps after complexation. Exothermic peaks registered in $320.54^{\circ} \mathrm{C}, 253.58^{\circ} \mathrm{C}$, and $281.4^{\circ} \mathrm{C}$ for $\mathrm{CH}, \mathrm{SA}$, and PEC nanoparticles, respectively, indicate that PEC nanoparticles have a peak value intermediate between the peaks of $\mathrm{SA}$ and $\mathrm{CH}$.

This peak value was interpreted as an interaction between the two polymers [24]. XRD spectra of PEG nanoparticles have shown the presence of 2 peaks of SA and a large bump. This indicates that SA was exposed on the outer surface and concealed the $\mathrm{CH}$ groups. The large bump indicates the entrapment of drug and formation of PEC (Fig. 3).

Two types of nanoparticles were prepared, i.e., PEC nanoparticles and $\mathrm{CH}$ nanoparticles. $\mathrm{CH}$ nanoparticles were only used for comparison, in in vitro and in vivo studies. Morphology of the PEC nanoparticles was determined by scanning electron microscopy (SEM) study. The shape of the PEC nanoparticles was found to be almost spherical (Fig. 4).

From the Table 1, it was observed that most of the alginate (AG), CH PEC nanoparticles have good \% entrapment efficiencies. With increase in concentration of $\mathrm{CH}$, the $\mathrm{EE}$ was increased. This was because of the increased number of complexes that are formed between $\mathrm{CH}$ and AG. The formulation AC6 has shown higher \% EE. One of the reasons behind this is the interaction of $\mathrm{CH}$ with $\mathrm{AG}$, and the other was the interaction of CH with LMWH which was negatively charged leading to higher EE. For obtaining a qualitative measure of the size of PECs, percentage transmittance studies were performed for formulations AC3 to AC9 at $800 \mathrm{~nm}$. At this wavelength, both AG and CH do not absorb any light [10]. Decrease in \% transmittance was a measure of increased particle size (Table 3). This may be because of the repulsion between the excess positive charges provided by the $\mathrm{CH}$ molecule in nanoparticles.

From the in vitro release studies, it was observed that $<1 \%$ of drug was released within 2 hrs in pH 1.2 buffer (Fig. 5). The complex was tough enough in the stomach because of the insolubility of AG at the particular $\mathrm{pH}$ [25]. In $\mathrm{pH} 6.8$ and $\mathrm{pH} 7.4$ buffer, a constant release of the LMWH was observed with a maximum release of $90 \%$ of the initial amount. $\mathrm{CH}$ nanoparticles have shown $31.45 \%$ release of the drug in $1.2 \mathrm{pH}$ buffer, which was not seen in PEC nanoparticles. The results of release kinetics revealed that the mode of drug release from PEC nanoparticles followed the Higuchi model with $\mathrm{r}^{2}>0.8654$ (Table 4). Data were also fitted into the KorsmeyerPeppas equation to determine the drug release mechanism further. The $\mathrm{n}$ value indicates super case-II transport [26]. Formulation AC3 was considered less stable as it has shown aggregation and increased particle size within 10 days. Taking into consideration EE, in vitro release the formulations AC4, AC5, AC7, and AC8 were evaluated for particle size, zeta potential, and polydispersity index (Table 5). Particle size was found to increase with increase in $\mathrm{CH}$ concentration. The zeta potential values have shown a shift in charge on the surface of nanoparticles from higher to lower negative values. Polydispersity

Table 1:Composition and \% EE of PEC nanoparticles

\begin{tabular}{|c|c|c|c|c|c|c|}
\hline Formulation & LMWH (mg) & CH (mg) & SA (mg) & Acetic acid $(\% \mathrm{v} / \mathrm{v})$ & Water (ml) & $\%$ EE \\
\hline AC1 & 20 & 100 & 100 & 1 & 100 & $45.5 \pm 2.65 \%$ \\
\hline AC2 & 20 & 200 & 100 & 1 & 100 & $50.5 \pm 2.73 \%$ \\
\hline AC3 & 20 & 200 & 250 & 1 & 100 & $77.25 \pm 0.8 \%$ \\
\hline AC4 & 20 & 300 & 150 & 1 & 100 & $72.26 \pm 1.1 \%$ \\
\hline AC5 & 20 & 300 & 250 & 1 & 100 & $84.88 \pm 0.87 \%$ \\
\hline AC6 & 20 & 300 & 400 & 1 & 100 & $88.15 \pm 0.9 \%$ \\
\hline AC7 & 20 & 350 & 100 & 1 & 100 & $67.43 \pm 0.48 \%$ \\
\hline AC9 & 20 & 400 & 300 & 1 & 100 & $87.26 \pm 0.7 \%$ \\
\hline AC10 & 20 & 500 & 100 & 1 & 100 & Viscous fibrous preparation \\
\hline AC11 & 20 & 500 & 200 & 1 & 100 & Viscous fibrous preparation \\
\hline AC12 & 20 & 500 & 300 & 1 & 100 & Viscous fibrous preparation \\
\hline
\end{tabular}

Data are expressed as mean \pm SD ( $n=3)$. SD: Standard deviation, LMWH: Low molecular weight heparin, CH: Chitosan, SA: Sodium alginate, EE: Entrapment efficiency,

PEC: Polyelectrolyte complexation 


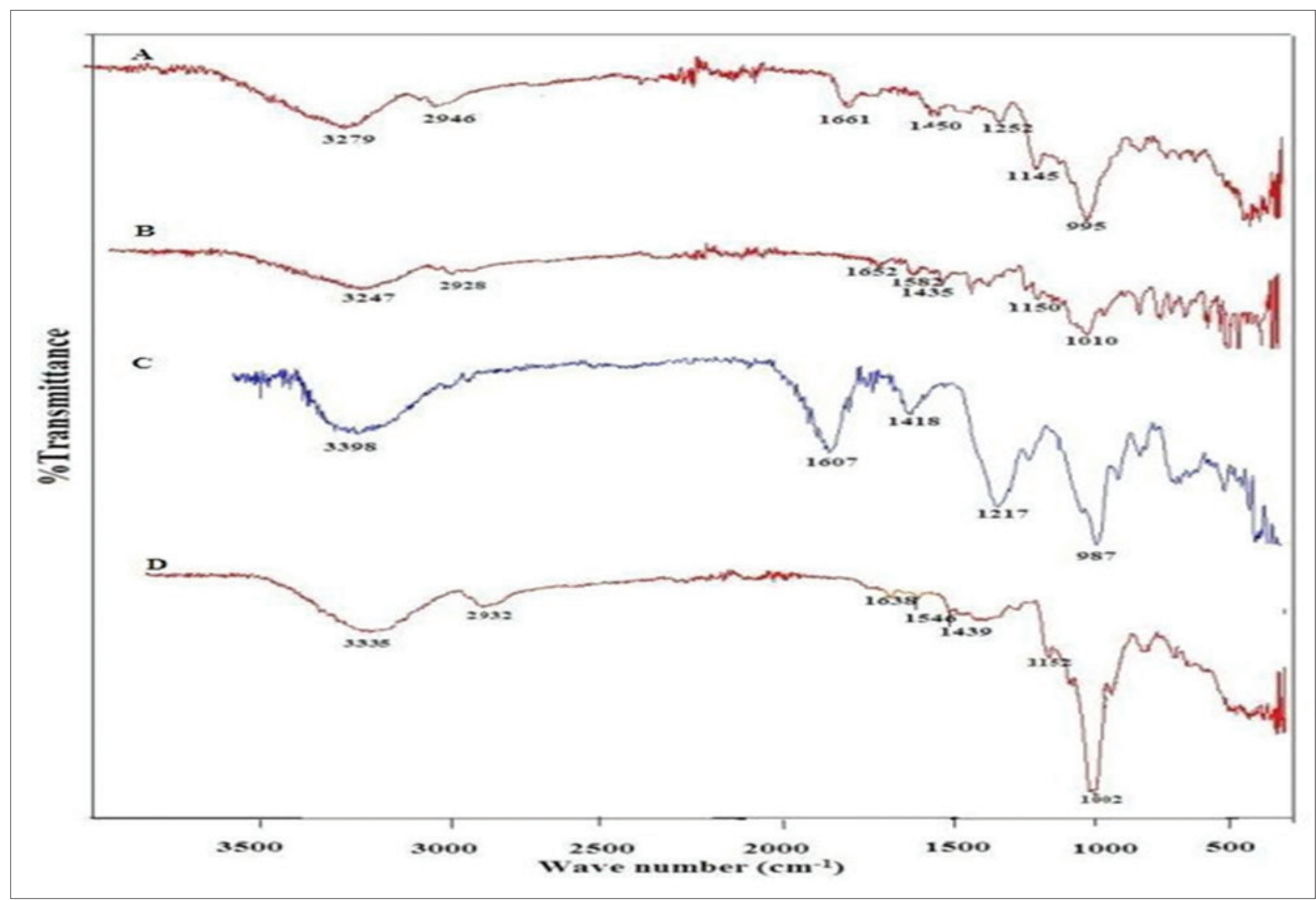

Fig. 1: Schematic diagram showing Fourier transform infrared spectra's of (a) Pure sodium alginate, (b) pure chitosan, (c) low-molecularweight heparin (LMWH), (d) polyelectrolyte complexed nanoparticles of LMWH

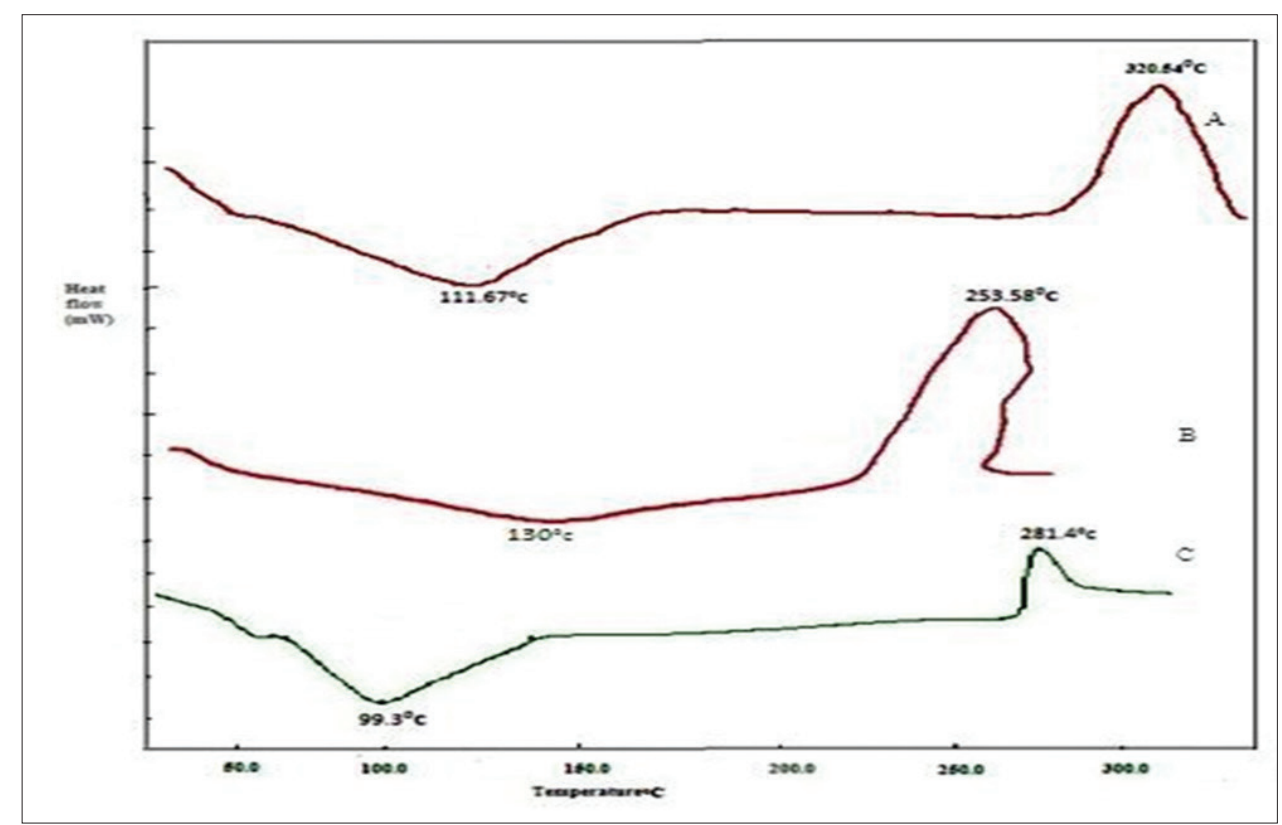

Fig. 2: Differential scanning calorimetry thermograms of (a) pure chitosan ( $\mathrm{CH})$, (b) pure sodium alginate, (c) polyelectrolyte complexed alginate/CH nanoparticles

index values $<0.5$ indicate that the formulation was homogeneous in nature.

In the results of ex vivo studies, $73 \%$ and $62 \%$ of the drug were found to cross the intestinal membrane for AC4 and AC5 formulations, respectively, and reach the buffer in 6 hrs time period (Fig. 6). The optimized nanoparticle formulation was subjected to stability studies at different storage temperatures in terms of particle size and EE. The nanoparticles showed better stability at $4^{\circ} \mathrm{C}$ than at $25^{\circ} \mathrm{C}$ in terms of increased particle size and decreased EE (Table 6). 


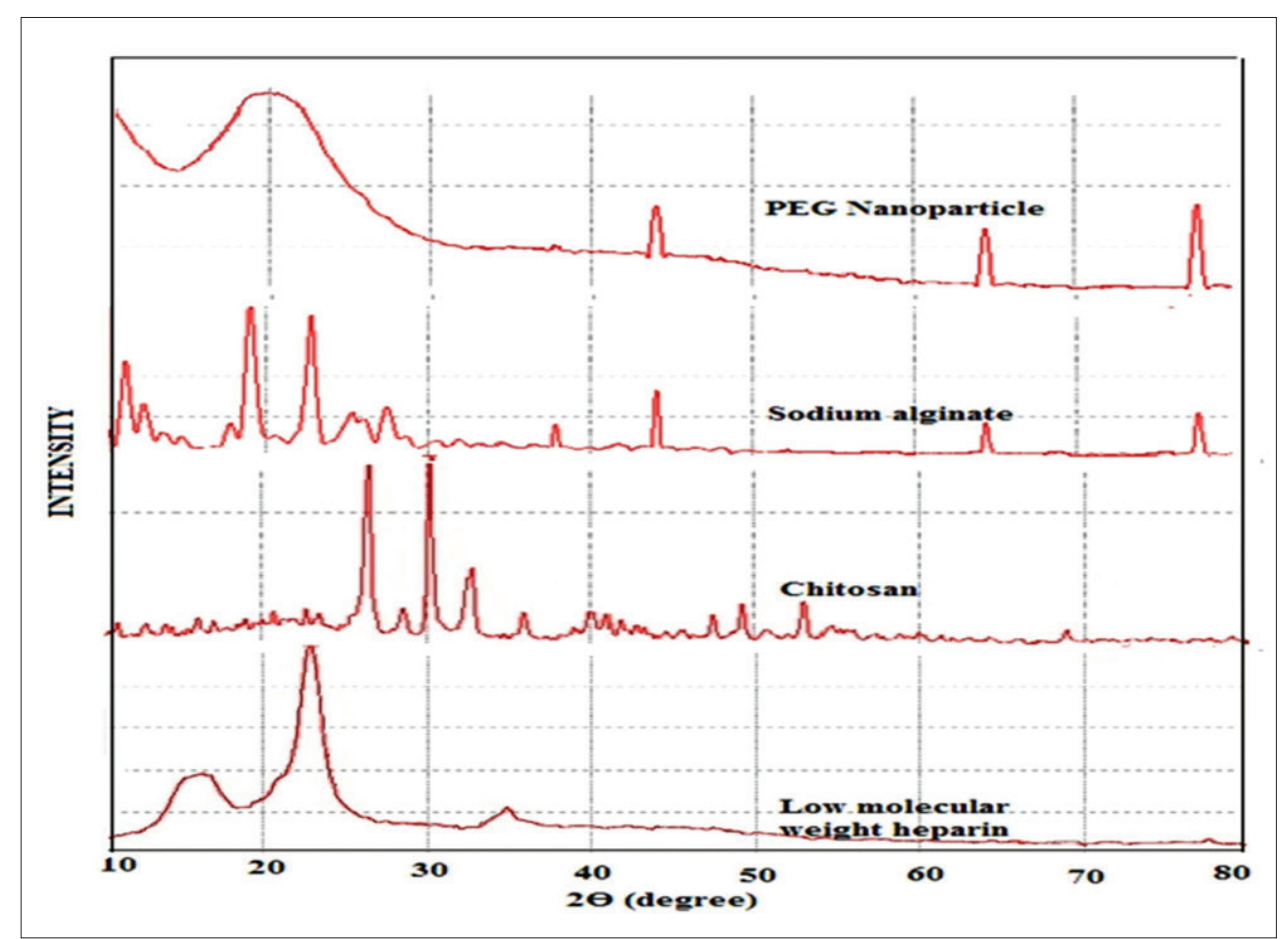

Fig. 3: X-ray diffraction patterns of polyelectrolyte complexation nanoparticles and its formulation ingredients

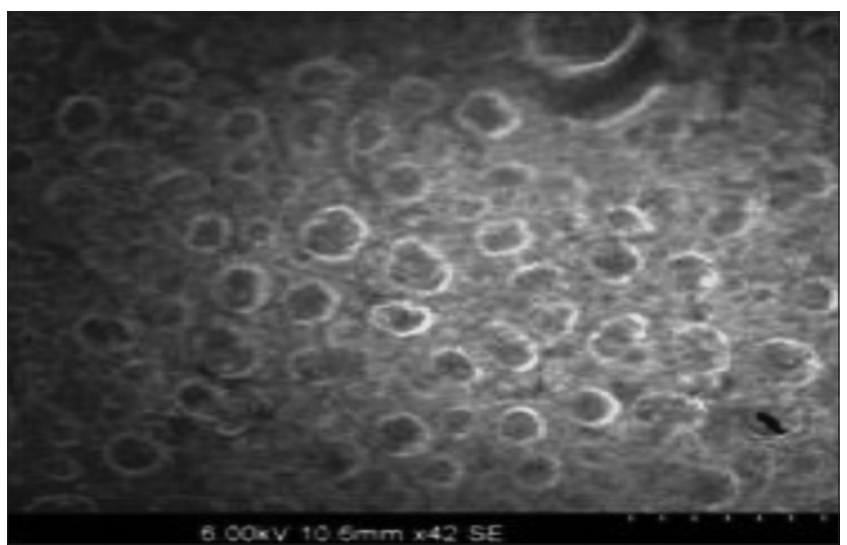

Fig. 4: Scanning electron microscopic micrographs of lowmolecular-weight heparin loaded polyelectrolyte complexation nanoparticles

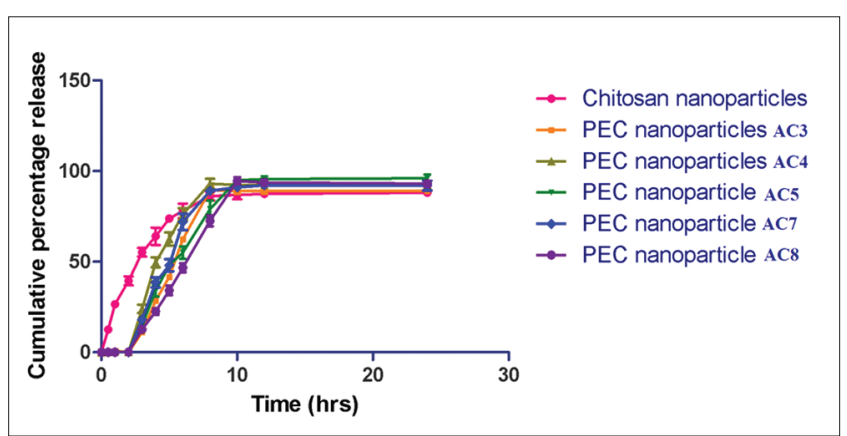

Fig. 5: In vitro release profile of low molecular weight heparin loaded polyelectrolyte complexation nanoparticles in different $\mathrm{pH}$ environments. Data represent mean \pm satndard deviation $(n=6)$

Pharmacokinetic parameters were determined from the plasma concentration-time profile of different formulations (Table 7).
Table 2: Important band frequencies in FTIR spectrum of drug, pure polymer, and nanoparticle

\begin{tabular}{ll}
\hline Wave number $\left(\mathbf{c m}^{-1}\right)$ & Interpretation \\
\hline Pure SA & \\
3279 & O-H stretch \\
2946 & C-H stretch \\
1661 & Asymmetric carboxylate anion (COO-) stretch \\
1450 & Symmetric carboxylate anion (COO-) stretch \\
1252 & C-O stretch \\
1145 & Bridge-O-stretch \\
997 & C-O-C stretch due to saccharide structure \\
Pure CH & \\
3247 & O-H stretch and N-H stretch \\
2928 & C-H stretch \\
1652 & Amide I NH CO stretch \\
1582 & Amide II N-H bend \\
1435 & C-N stretch \\
1150 & Bridge-O-stretch \\
1010 & C-O stretch \\
LMWH & \\
3398 & O-H stretch \\
1607 & N-H broadening \\
1418 & C-H stretch of methyl group \\
1217 & C-O stretch \\
987 & \\
PEC nanoparticles of & \\
LMWH & \\
3335 & O-H stretch \\
2932 & C-H stretch \\
1638 & Asymmetric carboxylate anion (COO-) tretch \\
1546 & Amide II N-H bend \\
1439 & Symmetric carboxylate anion (COO-) stretch \\
1152 & Bridge-O-stretch \\
1002 & C-O-C stretch \\
\hline & Din
\end{tabular}

LMWH: Low molecular weight heparin, CH: Chitosan, SA: Sodium alginate, PEC: Polyelectrolyte complexation, FTIR: Fourier transform infrared

For LMWH plain oral solution, the $\mathrm{C}_{\max }$ value was observed to be $0.10 \pm 0.007 \mathrm{IU} / \mathrm{mlafter} 2 \mathrm{hrs}$ of oral administration, while administration of $\mathrm{CH}$ nanoparticles enhanced the $\mathrm{C}_{\max }$ to $0.16 \pm 0.02 \mathrm{IU} / \mathrm{ml}$. However, 
Table 3: Percentage transmittance study of alginate/CH formulations

\begin{tabular}{llllllll}
\hline Alginate $/$ CH formulations & AC3 & AC4 & AC5 & AC6 & AC7 & AC8 & AC9 \\
\hline Percentage transmittance & $90 \pm 1.23 \%$ & $94 \pm 2.6 \%$ & $92 \pm 1.79 \%$ & $84 \pm 3.21 \%$ & $89 \pm 1.4 \%$ & $90 \pm 2.9 \%$ & $80 \pm 1.1 \%$ \\
\hline Data are expressed as mean \pm SD $(\mathrm{n}=3)$. SD: Standard deviation, CH: Chitosan & & & & &
\end{tabular}

Table 4: Comparison of estimated parameters deduced from in vitro LMWH release curve of PEC nanoparticles in mixed pH media by fitting to various kinetic models

\begin{tabular}{llllll}
\hline Formulation & Zero order & First order & Higuchi model & \multicolumn{2}{c}{ Hixon- crowell } \\
\cline { 2 - 5 } & $\mathbf{r}^{2}$ & $\mathbf{r}^{2}$ & $\mathbf{r}^{2}$ & $\mathbf{r}^{2}$ & 0.1753 \\
\hline AC3 & 0.4821 & 0.5692 & 0.8774 & 0.1759 & 0.9917 \\
AC4 & 0.3991 & 0.4054 & 0.8654 & 0.2862 & 0.9802 \\
AC5 & 0.5537 & 0.8772 & 0.9112 & 0.2862 & 0.9935 \\
AC7 & 0.6029 & 0.5524 & 0.9019 & 0.1926 & 0.9848 \\
AC8 & 0.5833 & 0.7472 & 0.8965 & 1.07 & 0.9903 \\
\hline
\end{tabular}

Data are expressed as mean \pm SD (n=3). SD: Standard deviation, LMWH: Low-molecular-weight heparin, PEC: Polyelectrolyte complexation

Table 5: Particle size, zeta potential, and polydispersity index of LMWH loaded PEC nanoparticles

\begin{tabular}{llll}
\hline Formulation & Particle size & Zetapotential & $\begin{array}{l}\text { Polydispersity } \\
\text { index }\end{array}$ \\
\hline AC4 & $119 \pm 1.3 \mathrm{~nm}$ & $-22 \pm 0.6$ & $0.25 \pm 0.02$ \\
AC5 & $142 \pm 0.8 \mathrm{~nm}$ & $-08 \pm 0.4$ & $0.33 \pm 0.03$ \\
AC7 & $195 \pm 0.3 \mathrm{~nm}$ & $-24 \pm 0.8$ & $0.41 \pm 0.01$ \\
AC8 & $253 \pm 2.5 \mathrm{~nm}$ & $-12 \pm 0.2$ & $0.11 \pm 0.06$ \\
\hline
\end{tabular}

Data are expressed as mean \pm SD $(n=3)$. SD: Standard deviation,

LMWH: Low-molecular-weight heparin, PEC: Polyelectrolyte complexation

the maximum value was observed for LMWH loaded PEC nanoparticles, which was $0.45 \pm 0.03 \mathrm{IU} / \mathrm{ml}$. Similarly, area under the curve $\mathrm{e}_{0.8 \mathrm{~h}}$ of LMWH was increased by 1.8 times for LMWH CH nanoparticles and 4.5 times for LMWH PEC nanoparticles when compared with a plain oral LMWH solution (Fig. 7). The results of clotting time studies have revealed an enhancement in clotting time (Fig. 8).

\section{DISCUSSION}

FTIR and DSC studies confirmed that the PEC was formed between AG and CH. DSC and XRD studies indicated that AG and $\mathrm{CH}$ were transformed to amorphous forms on complexation and LMWH was entrapped. SEM studies revealed that the formed nanoparticles were of spherical shape and the micrographs did not show the appearance of aggregated particles. Based on $\% \mathrm{EE}$ and $\%$ transmittance studies, the formulations AC4, AC5, AC6, AC7, and AC8 were optimized. In vitro release studies indicate that PEC nanoparticles were successful in retarding the release of drug in acidic $\mathrm{pH}$ conditions and capable of releasing $90 \%$ of the drug in simulated intestinal fluid within $10 \mathrm{hrs}$ time period. This release of LMWH in small intestine could be because of the interaction of AG with alkaline media and an increase in the solubility of AG [27].

The possible mechanism of drug release from LMWH PEC nanoparticles was found to be super case-II transport, which was considered as swelling-controlled release. It was observed that with an increase in concentration of $\mathrm{CH}$ the particle size was increased and the zeta potential value has shifted toward lower negative charges. The negative charge demonstrates that AG has formed complex with $\mathrm{CH}$ and helped in concealing $\mathrm{CH}$ from degradation in the gastric environment. Ex vivo permeation studies indicated that the enhanced transport of PEC nanoparticles was ascribed to the mucoadhesive property of $\mathrm{CH}$ and the size of nanoparticles [28].

One of the research articles demonstrated that LMWH loaded trimethyl $\mathrm{CH}$ nanoparticles have shown 2.4 times bioavailability of LMWH in comparison to plain LMWH solution [19]. Pharmacokinetic studies of the present study have shown an enhancement in oral

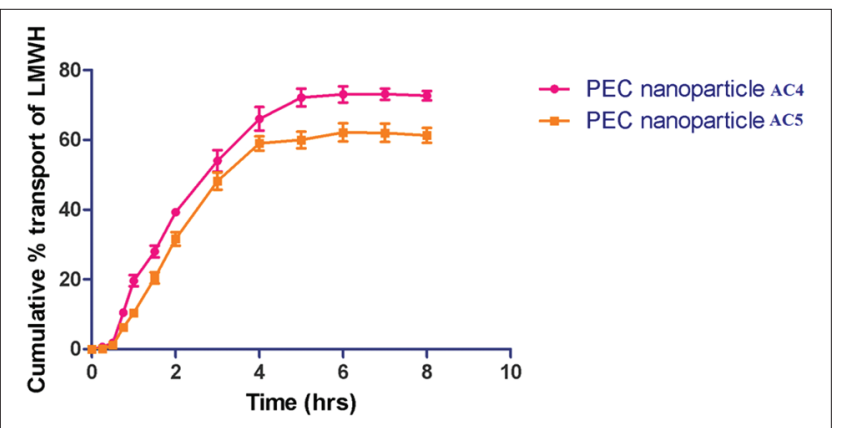

Fig. 6: Cumulative percent transport of low molecular weight heparin from polyelectrolyte complexation nanoparticles AC4 and AC5. Data represent mean \pm standard deviation $(n=6)$

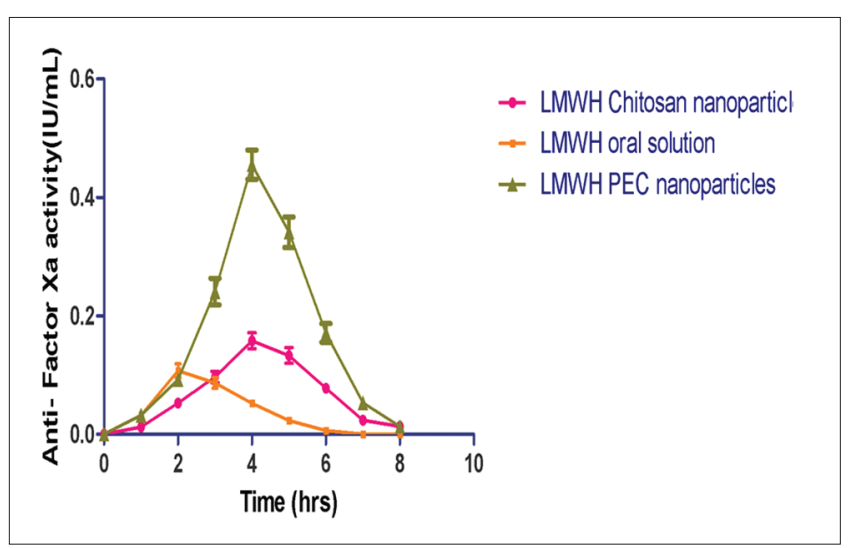

Fig. 7: Antifactor Xa activity profile of low-molecular-weight heparin (LMWH) oral solution, LMWH chitosan nanoparticles, and LMWH polyelectrolyte complexation nanoparticles after oral administration in equivalent dose of $50 \mathrm{mg} / \mathrm{kg}$ in Wistar rats. Data represent mean \pm standard deviation $(n=6)$

bioavailability of LMWH by 4.5 times when compared with a plain LMWH solution. This enhanced oral bioavailability was attributed to the interaction of $\mathrm{AG}$ and $\mathrm{CH}$, which prevented the degradation of drug in gastric fluid. PEC nanoparticles then enter the small intestine where the AG gets slowly dissolved exposing the amino groups of $\mathrm{CH}$. These amino groups get attached to the negative charge of epithelial lining and help with paracellular transport across the intestinal epithelium. This results in delivery of the drug to systemic circulation without damaging the intestinal epithelium. The in vitro and in vivo evaluation of PEC nanoparticles demonstrates that the 
Table 6: Effect of storage temperature on particle size and EE of LMWH loaded PEC nanoparticles

\begin{tabular}{|c|c|c|c|c|c|c|}
\hline \multirow[t]{3}{*}{ Formulation } & \multicolumn{3}{|l|}{ Particle size (nm) } & \multicolumn{3}{|l|}{ EE (\%) } \\
\hline & \multirow{2}{*}{$\begin{array}{l}0 \text { day } \\
\text { Room temperature }\end{array}$} & \multicolumn{2}{|c|}{ After 60 days } & \multirow{2}{*}{$\begin{array}{l}\mathbf{0} \text { day } \\
\text { Room temperature }\end{array}$} & \multicolumn{2}{|c|}{ After 60 days } \\
\hline & & $4 \pm 1^{\circ} \mathrm{C}$ & $25 \pm 1^{\circ} \mathrm{C}$ & & $4 \pm 1^{\circ} \mathrm{C}$ & $25 \pm 1^{\circ} \mathrm{C}$ \\
\hline PEC nanoparticle & $105 \pm 1.3$ & $119 \pm 3.9$ & $276 \pm 2.6$ & $80 \pm 3.4$ & $74 \pm 2.1$ & $66 \pm 4.2$ \\
\hline
\end{tabular}

Data are expressed as mean \pm SD (n=3). SD: Standard deviation, LMWH: Low-molecular-weight heparin, PEC: Polyelectrolyte complexation, EE: Entrapment efficiency

Table 7: Pharmacokinetic parameters of LMWH nanoparticles after oral administration in Wistar rats

\begin{tabular}{llll}
\hline Pharmacokinetic parameters & LMWH oral solution & LMWH CH nanoparticle & LMWH PEC nanoparticle \\
\hline Dose $(\mathrm{mg})$ & 10 & 10 & 10 \\
$\mathrm{C}_{\max }(\mathrm{IU} / \mathrm{ml})$ & $0.10 \pm 0.007$ & $0.16 \pm 0.02$ & $0.45 \pm 0.03$ \\
$\mathrm{~T}_{\max }(\mathrm{h})$ & 2 & 4 & 4 \\
$\mathrm{AUC}_{0-8 \mathrm{~h}}(\mu \mathrm{g} \mathrm{h} / \mathrm{ml})$ & $0.31 \pm 0.006$ & $0.56 \pm 0.04$ & $1.397 \pm 0.24$ \\
$\left.\mathrm{MRT}^{\mathrm{h}}\right)$ & $2.81 \pm 0.014$ & $4.24 \pm 0.012$ & \\
$\mathrm{t}_{\text {half }}(\mathrm{hr})$ & $0.56 \pm 0.002$ & $0.72 \pm 0.03$ & $4.36 \pm 0.11$ \\
\hline
\end{tabular}

Data are expressed as mean \pm SD ( $n=6)$. MRT: Mean residence time, AUC: Area under the curve, LMWH: Low-molecular-weight heparin, PEC: Polyelectrolyte complexation, $\mathrm{CH}$ : Chitosan

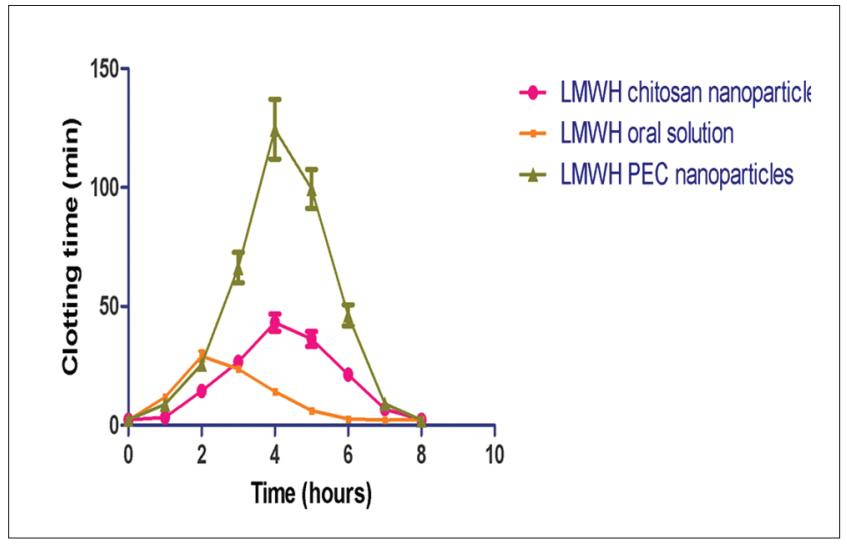

Fig. 8: Clotting time profile of low molecular weight heparin (LMWH) oral solution, LMWH chitosan nanoparticles and LMWH polyelectrolyte complexation nanoparticles after oral administration in equivalent dose of $50 \mathrm{mg} / \mathrm{kg}$ in wistar rats. Data represents mean \pm standard deviation $(n=6)$

nanoparticulate system can be considered as a useful oral delivery system for enhancing the bioavailability of LMWH.

\section{CONCLUSION}

The approach to the oral delivery of LMWH is of utmost importance to prevent the invasive delivery. $\mathrm{CH}$ nanoparticles can be considered for oral delivery, but the high solubility of $\mathrm{CH}$ in acidic $\mathrm{pH}$ conditions makes it less suitable for oral administration. Hence, PEC nanoparticles were developed with $\mathrm{AG}$ and $\mathrm{CH}$ to overcome the limitations associated with plain CH nanoparticles. The developed PEC nanoparticles have shown an enhanced oral bioavailability in comparison with a plain LMWH solution. The present investigation suggests that the AG complexed $\mathrm{CH}$ nanoparticles could be effectively explored for the oral delivery of LMWH.

\section{ACKNOWLEDGMENTS}

The authors acknowledge the Principal and Management of Vaagdevi College of Pharmacy, Hanamkonda, Sri Padmavathi Mahila Vishwavidhyalayam, Tirupati, for providing the necessary facilities helpful in the conduct of the work.

\section{REFERENCES}

1. Andersson M, Löfroth JE. Small particles of a heparin/chitosan complex prepared from a pharmaceutically acceptable microemulsion. Int J Pharm 2003;257(1-2):305-9.

2. Wang J, Tan H, Yu A, Ling P, Lou H, Zhai G, et al. Preparation of chitosan-based nanoparticles for delivery of low molecular weight heparin. J Biomed Nanotechnol 2011;7(5):696-703.

3. Aysu Y, Ferhan S. An overview of modified release chitosan, alginate and Eudragit RS microparticles. J Chem Pharm Res 2010;2(3):704-21.

4. Scala-Bertola J, Gajdziok J, Rabisková M, Bonneaux F, Lecompte T, Sapin A, et al. Pellets for oral administration of low-molecular-weight heparin. Drug Dev Ind Pharm 2009;35(12):1503-10.

5. Kim SK, Vaishali B, Lee E, Lee S, Lee YK, Kumar TS, et al. Oral delivery of chemical conjugates of heparin and deoxycholic acid in aqueous formulation. Thromb Res 2006;117(4):419-27.

6. Lin IC, Liang M, Liu TY, Monteiro MJ, Toth I. Cellular transport pathways of polymer coated gold nanoparticles. Nanomedicine 2012;8(1):8-11.

7. Jogala S, Rachamalla SS, Aukunuru J. Development of subcutaneous sustained release nanoparticles encapsulating low molecular weight heparin. J Adv Pharm Technol Res 2015;6(2):58-64.

8. Yang T, Nyiawung D, Silber A, Hao J, Lai L, Bai S. Comparative studies on chitosan and polylactic-co-glycolic acid incorporated nanoparticles of low molecular weight heparin. AAPS PharmSciTech 2012;13(4):1309-18.

9. Bigucci F, Luppi B, Cerchiara T, Sorrenti M, Bettinetti G, Rodriguez L, et al. Chitosan/pectin polyelectrolyte complexes: Selection of suitable preparative conditions for colon-specific delivery of vancomycin. Eur J Pharm Sci 2008;35(5):435-41.

10. Saether HV, Holme HK, Maurstad G, Smidsrod O, Stokke BT. Polyelectrolyte complex formation using alginate and chitosan. Carbohydr Polym 2008; 74:813-21.

11. Liao IC, Wan AC, Yim EK, Leong KW. Controlled release from fibers of polyelectrolyte complexes. J Control Release 2005;104:347-58.

12. Hamman JH. Chitosan based polyelectrolyte complexes as potential carrier materials in drug delivery systems. Mar Drugs 2010;8:1305-22.

13. Cheung RC, Ng TB, Wong JH, Chan WY. Chitosan: An update on potential biomedical and pharmaceutical applications. Mar Drugs 2015;13(8):5156-86.

14. Li X, Xie H, Lin J, Xie W, Maa X. Characterization and biodegradation of chitosan-alginate polyelectrolyte complexes. Polym Degrad Stabil 2009;94:1-6.

15. Han T, New N, Furuike T, Tokura S, Tamura H. Methods of N-acetylated chitosan scaffolds and it's in vitro biodegradation by lysozyme. J Biomed Sci Eng 2012;5:15-23.

16. Aiba S. Studies on chitosan: 4. Lysozymic hydrolysis of partially $\mathrm{N}$-acetylated chitosans. Int J Biol Macromol 1992;14(4):225-8. 
17. Hoffart V, Ubrich B, Simonin C, Babak V, Vigneron C, Hoffman M, et al. Low molecular weight heparin-loaded polymeric nanoparticles: Formulation characterization and release characteristics. Drug Dev Ind Pharm 2002;28(9):1091-9.

18. Bagre AP, Jain K, Jain NK. Alginate coated chitosan core shell nanoparticles for oral delivery of enoxaparin: In vitro and in vivo assessment. Int J Pharm 2013;456(1):31-40.

19. Paliwal R, Paliwal SR, Agrawal GP, Vyas SP. Chitosan nanoconstructs for improved oral delivery of low molecular weight heparin: In vitro and in vivo evaluation. Int J Pharm 2012;422(1-2):179-84.

20. Deepak SN, Veda Hari BN. Optimization, development and evaluation of microemulsion for the release of combination of guaifenesin and phenylephrine. J Appl Pharm Sci 2013;3(9):48-56.

21. Ganeshkumar M, Ponrasu T, Sathishkumar M, Suguna L. Preparation of amphiphilic hollow carbon nanosphere loaded insulin for oral delivery. Colloids Surf B Biointerfaces 2013;103:238-43.

22. García-Manzano A, González-Llaven J, Lemini C, Rubio-Póo C. Standardization of rat blood clotting tests with reagents used for humans. Proc West Pharmacol Soc 2001;44:153-5.

23. Lemini C, Rubio-Póo C, Silva G, García-Mondragón J, Zavala E, Mendoza-Patiño $\mathrm{N}$, et al. Anticoagulant and estrogenic effects of two new 17 beta-aminoestrogens, butolame [17 beta-(4-hydroxy1-butylamino)-1,3,5(10)-estratrien-3-ol] and pentolame [17 beta(5-hydroxy-1-pentylamino)-1,3,5(10)-estratrien-3-ol]. Steroids 1993;58:457-61.

24. Rafie A, Alimohammadian MH, Gazori T, Riazi-rad F, Fatemi SM, Parizadeh A, et al. Comparison of chitosan, alginate and chitosan/ alginate nanoparticles with respect to their size, stability, toxicity and transfection. Asian Pac J Trop Dis 2014;4(5):372-7.

25. Sarmento B, Ribeiro A, Veiga F, Sampaio P, Neufeld R, Ferreira D. Alginate/chitosan nanoparticles are effective for oral insulin delivery. Pharm Res 2007;24(12):2198-206.

26. Ilango KB, Kavimani S. A systematic review of mathematical models of pharmaceutical dosage forms. Int J Curr Pharm Rev Res 2015;6(1):59-70.

27. Mukhopadhyay P, Chakraborty S, Bhattacharya S, Mishra R, Kundu PP. $\mathrm{pH}$-sensitive chitosan/alginate core-shell nanoparticles for efficient and safe oral insulin delivery. Int J Biol Macromol 2015;72:640-8.

28. Sonaje K, Lin YH, Juang JH, Wey SP, Chen CT, Sung HW. In vivo evaluation of safety and efficacy of self-assembled nanoparticles for oral insulin delivery. Biomaterials 2009;30(12):2329-39. 